\title{
Unitarity approach to the mass-dependent fit of $3 \pi$ resonance production data from the COMPASS experiment
}

\author{
Mikhail Mikhasenko ${ }^{1, a}$, Andrew Jackura², Bernhard Ketzer ${ }^{1}$, and Adam Szczepaniak ${ }^{2,3}$ \\ for the COMPASS Collaboration and the JPAC Collaboration \\ ${ }^{1}$ Universität Bonn, Helmholtz-Institut für Strahlen- und Kernphysik, 53115 Bonn, Germany \\ ${ }^{2}$ Indiana University, Bloomington IN, U.S.A. \\ ${ }^{3}$ Thomas Jefferson National Accelerator Facility, Newport News VA, U.S.A.
}

\begin{abstract}
We derive a unitarized model for the peripheral production of the three-pion system in the isobar approximation. The production process takes into account longrange $t$-channel pion exchange. The K-matrix approach is chosen for the parameterization of the scattering amplitude. Five coupled channels are used to fit the COMPASS spin-density matrices for the $J^{P C} M^{\epsilon}=2^{-+} 0^{+}$sector. Preliminary results of the fit are presented.
\end{abstract}

\section{Introduction}

More than one hundred mesons observed in the light sector are coupled to the system of three pions. Apart from being the lightest system of three particles, it can also have any combination of quantum numbers $J^{P C}$. Since a long time the exotic sector $1^{-+}$attracts the attention of theoretical and experimental groups. But also the interactions in the ordinary $J^{P C}$ sectors e.g. $1^{++}, 2^{++}, 2^{-+}$are very interesting as many resonances are known to appear in the scattering. Recently, the interest in hadron resonances has been growing due to several reasons. First, the calculation of scattering processes from first principles became possible using lattice techniques [1,2]. The extraction of observables from lattice data requires a theoretical framework similar to the one needed for real data. Second, modern experiments have collected unprecedentedly huge data samples making it possible to distinguish tiny features of strong interactions. Precise studies of the scattering amplitudes and resonance phenomena will eventually lead to a better understanding of Quantum Chromodynamics (QCD). Third, the understanding of hadronic background is very important for measurements of multiplicities and asymmetries. The available parameterizations of meson interaction amplitudes will improve Monte-Carlo generators.

The goal of our analysis is to develop the theoretical framework for the extraction of scattering amplitudes from different experimental data on meson scattering [3]. The scattering amplitude being an analytic function allows for continuation to the complex plane and the search for poles in this plane.

The difficulty is that at the energy range of meson resonances, reactions can not be constrained by effective theories using perturbative methods. The unitarity of the scattering matrix becomes one of

ae-mail: mikhail.mikhasenko@gmail.com 
the most important constraints to the scattering amplitude. The second assumption we will make is analyticity of the amplitude in the space of invariants upon which the amplitude depends.

\section{Peripheral production}

One way to study hadron spectroscopy is a reaction in a fixed-target experimental setup. The production of a hadronic system by the scattering of a high-energy beam particle with a small transferred momentum is called peripheral production. Several approximations for the amplitude of the reaction can be made due to the specific kinematics. $s$-channel resonance contributions become negligible due to the high total invariant mass. Thus, $t$-channel exchange is the dominant production mechanism. The reaction can be viewed as the scattering of the beam particle and the exchange particle.

An example of a recent high-statistics data sample for peripheral production is the reaction

$$
\pi^{-} p \rightarrow \pi^{-} \pi^{+} \pi^{-} p
$$

from the COMPASS experiment [4]. The pion beam momentum is $190 \mathrm{GeV}$, which guarantees peripheral scattering. A dedicated trigger for the recoil proton enables to select scattering events off protons. Since natural exchanges are allowed in the $t$-channel, the dominating production process is Pomeron exchange. In our approach, the Pomeron is not a particle with fixed quantum numbers but a trajectory which determines the behavior of the amplitude as a function of $s_{\text {tot }}$ and $t$.

The data sample for the production of charged three pions in the COMPASS experiment consists of approximately $5 \times 10^{6}$ events [5]. The distribution of the invariant mass of the $3 \pi$ system shows the presence of many resonances in the region $1-3 \mathrm{GeV}$. The distribution of the events over $t^{\prime}$ exhibits a sharp peak towards small values of $t^{\prime}$. The Dalitz plots for narrow intervals of the $3 \pi$ invariant mass indicate the presence of several resonances in $\pi^{+} \pi^{-}$-subsystem.

\subsection{Partial-wave expansion and spin-density matrix}

In this section we introduce the notation and clarify the quantities which are used to represent the results of the COMPASS partial-wave analysis (PWA). In general, the amplitude for the reaction $A+B \rightarrow 1+2+3+D$ depends on 8 variables. One common choice is the set $\kappa=\left(s_{\text {tot }}, s, t, s_{1}, \Omega_{1}, \Omega_{23}\right)$, with the total invariant mass of the reaction $s_{\text {tot }}=\left(p_{A}+p_{B}\right)^{2}$, the transferred momentum $t=\left(p_{B}-p_{D}\right)^{2}$ and $t^{\prime}=|t|-|t|_{\text {min }}$, the invariant mass of the three-pion system $s=\left(p_{1}+p_{2}+p_{3}\right)^{2}$, the invariant mass of one of the pion pairs $s_{1}=\left(p_{2}+p_{3}\right)^{2}$, the two angles of the vector $\vec{p}_{2}+\vec{p}_{3}$ in the Gottfried-Jackson (GJ)-frame $\Omega_{1}[6]$ and the two angles of the second pion $\overrightarrow{p_{2}}$ in the rest-frame of the selected pion pair, obtained from the GJ-frame by sequential rotation and boost, $\Omega_{23}$.

A mass-independent partial-wave analysis of the $3 \pi$ data set has been performed by COMPASS [5]. In this analysis, the amplitude is expanded in a truncated set of partial waves. A partial wave is defined by its quantum numbers: the total angular momentum $J$ and its projection to the $z$-axis in the GJ frame $M$, and the reflectivity quantum number $\epsilon$ which corresponds to the naturality of the exchanged particle in the $t$-channel. A pair of pions chosen for the expansion is projected to state with spin $S$. The $\pi \pi$-state is called isobar state. The orbital angular momentum between the isobar and the bachelor pion is denoted as $L$.

The partial-wave amplitude includes an angular part, which factorizes out the dependence on $\Omega_{1}$ and $\Omega_{23}$. The expansion coefficients generally depend on the four variables $s_{\text {tot }}, s, t, s_{1}$. In the approximation of the Isobar Model, the whole dynamics in $s_{1}$ is assumed to be contained in the isobar amplitude $f_{S}\left(s_{1}\right)$ :

$$
A_{1}(\kappa)=\sum_{J M \epsilon L S}\left(\hat{F}_{1}\right)_{L S}^{J M \epsilon}\left(s_{\mathrm{tot}}, t, s\right) f_{S}\left(s_{1}\right)\left(Z_{1}\right)_{L S}^{J M \epsilon}\left(\Omega_{1}, \Omega_{23}\right)
$$


where the angular basis functions are obtained from the recoupling of the helicity basis functions [7]. The functions $\left(Z_{1}\right)_{L S}^{J M \epsilon}$ are orthogonal and normalized to unity. The orthogonality in $L$ is ensured by the properties of the Clebsch-Gordan coefficients $\langle L O S \lambda \mid J \lambda\rangle$.

$$
\left(Z_{1}\right)_{L S}^{J M \epsilon}\left(\Omega_{1}, \Omega_{23}\right)=\left(\frac{2 L+1}{2 J+1}\right)^{1 / 2} \sum_{\lambda}\langle L 0 S \lambda \mid J \lambda\rangle\left(\frac{2 J+1}{4 \pi}\right)^{1 / 2} D_{M \lambda}^{J \epsilon *}\left(\Omega_{1}\right)\left(\frac{2 S+1}{4 \pi}\right)^{1 / 2} D_{\lambda 0}^{* S}\left(\Omega_{23}\right) .
$$

The reflectivity basis of the Wigner $D$-functions is used $[8,9]$ :

$$
D_{M \lambda}^{J \epsilon}(\Omega)=\theta(M)\left[D_{M \lambda}^{J}(\Omega)-\epsilon(-)^{J-M} D_{-M \lambda}^{J}(\Omega)\right],
$$

where $\theta(M)$ is zero for negative $M$, while it is equal to $1 / 2$ for $M=0$ and $1 / \sqrt{2}$ for positive $M$. Wigner $D_{m_{1} m_{2}}^{J}$-functions of two angles $\Omega=(\theta, \phi)$ are defined as $D_{m_{1} m_{2}}^{J}(\Omega)=D_{m_{1} m_{2}}^{J}(\phi, \theta, 0)$.

The presence of identical particles in the final state implies a symmetry of the amplitude under permutations of $\pi^{-}$. The symmetrization is done explicitly. ${ }^{1}$ When the order of pions is fixed to be $\pi^{-} \pi^{+} \pi^{-}$we have

$$
A(\kappa)=\left(A_{1}+A_{3}\right) / \sqrt{2},
$$

where the factor $1 / \sqrt{2}$ has been moved to the amplitude from phase space integration.

In the isobar model the amplitude $\hat{F}_{L S}^{J M \epsilon}$ is common for both subchannel partial waves, thus it can be pulled out. We group the fully symmetrized partial-wave amplitude to the fraction term:

$$
A=\sum_{J M \epsilon L S} \hat{F}_{L S}^{J M \epsilon}\left(s_{\mathrm{tot}}, t, s\right) \frac{\left(Z_{1}\right)_{L S}^{J M \epsilon} f_{S}\left(s_{1}\right)+\left(Z_{3}\right)_{L S}^{J M \epsilon} f_{S}\left(s_{3}\right)}{\sqrt{2}} .
$$

The square of the amplitude integrated over three-body phase space becomes proportional to the number of events observed in a selected narrow interval of $3 \pi$ invariant mass and $t^{\prime}$. We introduce the notation $\varrho_{L S L^{\prime} S^{\prime}}^{J M \epsilon}=\hat{F}_{L S}^{J M \epsilon} \hat{F}_{L^{\prime} S^{\prime}}^{* J \epsilon}$, then $\mathrm{d}^{2} N /(\mathrm{d} s \mathrm{~d} t)=N_{0} \varrho_{L S L^{\prime} S^{\prime}}^{J M \epsilon} \Phi_{L S L^{\prime} S^{\prime}}^{J M \epsilon}$, where the sum over $J, M, \epsilon, L, S, L, S^{\prime}$ is assumed, $N_{0}$ is a normalization constant, and

$$
\Phi_{L S L^{\prime} S^{\prime}}^{J M \epsilon}=\frac{1}{2} \int \mathrm{d} \Phi_{3 \pi}\left[\left(Z_{1}\right)_{L S}^{J M \epsilon} f_{S}\left(s_{1}\right)+\left(Z_{3}\right)_{L S}^{J M \epsilon} f_{S}\left(s_{3}\right)\right]\left[\left(Z_{1}\right)_{L^{\prime} S^{\prime}}^{J M \epsilon} f_{S}\left(s_{1}\right)+\left(Z_{3}\right)_{L^{\prime} S^{\prime}}^{J M \epsilon} f_{S}\left(s_{3}\right)\right]^{\star}
$$

It is convenient to normalize the spin-density matrix to the number of events,

$$
\stackrel{\circ}{\Phi}_{L S L^{\prime} S^{\prime}}^{J M \epsilon}=\Phi_{L S L^{\prime} S^{\prime}}^{J M \epsilon} /\left(\Phi_{L S}^{J M \epsilon} \Phi_{L^{\prime} S^{\prime}}^{J M \epsilon}\right)^{1 / 2}, \quad{\stackrel{\varrho}{L S L^{\prime} S^{\prime}}}_{U M \epsilon}=N_{0} \varrho_{L S L^{\prime} S^{\prime}}^{J M \epsilon}\left(\Phi_{L S}^{J M \epsilon} \Phi_{L^{\prime} S^{\prime}}^{J M \epsilon}\right)^{1 / 2}
$$

$\Phi_{L S}^{J M \epsilon}$ denotes the diagonal part of the matrix $\Phi_{L S}^{J M \epsilon}=\Phi_{L S L S}^{J M \epsilon}$. We thus have arrived at the expression

$$
\mathrm{d}^{2} N /(\mathrm{d} s \mathrm{~d} t)=\varrho_{L S L^{\prime} S^{\prime}}^{J M \epsilon}, \stackrel{\Phi}{\Phi}_{L S L^{\prime} S^{\prime}}^{J M \epsilon}
$$

where the sum over all indices is implicitly assumed. Partial-wave intensities and relative phases are defined from the spin-density matrix $\varrho_{L S L^{\prime} S^{\prime}}^{J M \epsilon}$ as diagonal elements and arguments of the off-diagonal elements, respectively.

\footnotetext{
${ }^{1}$ We note that the symmetrized amplitude for a selected partial wave does not have fixed $L S$ quantum numbers. The basis functions $\left(Z_{1}\right)_{L S}^{J M \epsilon}$ and $\left(Z_{3}\right)_{L^{\prime} S^{\prime}}^{J M \epsilon}$ are not orthogonal.
} 


\section{The model with unitarity constraints}

Peripheral production suggests that the target recoil particle is kinematically decoupled from the fast moving $3 \pi$ system. The reaction can thus be considered as

$$
\pi \mathbb{P} \rightarrow \pi^{-} \pi^{+} \pi^{-}
$$

From angular momentum conservation the magnetic quantum number $M$ is equal to minus the helicity of the Pomeron measured in the $3 \pi$ rest-frame. ${ }^{2}$

The amplitude for the reaction (10) is denoted as $A$, i.e. $\langle 3 \pi|\mathbb{T}| \pi \mathbb{P}\rangle=(2 \pi)^{4} \delta\left(P_{A \mathbb{P}}-P\right) A$. The $S$-matrix operator $\mathbb{S}$ is connected to the interaction operator $\mathbb{T}$ by $\mathbb{S}=\mathbb{I}+i \mathbb{T}$. The unitarity constraint $\mathbb{S}^{\dagger} \mathbb{S}=\mathbb{I}$ is applied to reaction (10):

$$
\left\langle 3 \pi\left|\mathbb{T}-\mathbb{T}^{\dagger}\right| \pi \mathbb{P}\right\rangle=i\left\langle 3 \pi\left|\mathbb{T}^{\dagger} \mathbb{T}\right| \pi \mathbb{P}\right\rangle .
$$

To proceed the identity operator is substituted between $\mathbb{T}^{\dagger}$ and $\mathbb{T}$. The operator has a sum/integral over different states and possible momentum configurations,

$$
\mathbb{I}=\sum_{\text {momenta }}|3 \pi\rangle\langle 3 \pi|+\ldots
$$

Simplification 1: we take into account only elastic contributions to the intermediate states. For the present application of the model, the quantum numbers of the final state will be restricted to $2^{-+}$ where, indeed, three-pion intermediate states contribute the most. For the extension of the model to other cases, e.g. $2^{++}$, other intermediate states $(\eta \pi, K \bar{K} \pi)$ have to be included.

Simplification 2: we approximate the $3 \pi$ system by the isobar-pion system and neglect cross terms in the rescattering. When the cross terms in Eq. 7 are dropped for consistency, the matrix becomes diagonal. The state $|3 \pi\rangle$ under the approximation is denoted as $|I \pi\rangle$. For concreteness, the isobar is set to be in channel $\pi_{2} \pi_{3}$. We drop the index 1 for $\left(F_{1}\right)_{L S}^{J M \epsilon}$ and $\left(Z_{1}\right)_{L S}^{J M \epsilon}$ in the further discussion.

Time reversal symmetry is used to relate $\left\langle f\left|\mathbb{T}^{\dagger}\right| i\right\rangle$ to complex conjugated matrix element, $\langle f|\mathbb{T}| i\rangle^{\star}$, in Eq. 11.

$$
A-A^{\star}=i \int \mathrm{d} \Phi_{3} T^{\star} A, \quad\left\langle I^{\prime} \pi|\mathbb{T}| I \pi\right\rangle=(2 \pi)^{4} \delta\left(P^{\prime}-P\right) T .
$$

We introduce the amplitude $T$ as the transition amplitude $I^{\prime} \pi \rightarrow I \pi$. The quantity describes the interaction of the system of three pions. $T$ is a production process-independent quantity, but it can not be measured directly. The amplitude for the full reaction has to be related to $T$. The amplitude for three-to-three scattering depends on 8 variables. With our isobar approximation, we parametrize the isobar production and decay from both sides. The variables are as follows: invariant mass of the system $s$, variables related to initial state $s_{1}, \Omega_{1}, \Omega_{23}$, variables related to the final state $s_{1}^{\prime}, \Omega_{1}^{\prime}, \Omega_{23}^{\prime}$. The production plane for the initial and final state can be chosen arbitrarily, so from $\Omega_{1}, \Omega_{1}^{\prime}$ there is only one polar angle which the amplitude depends on.

A partial-wave expansion is performed for $T$ from both sides

$$
T=Z_{L S}^{* J M \epsilon}\left(\Omega_{1}, \Omega_{23}\right) T_{L S L^{\prime} S^{\prime}}^{J M \epsilon}\left(s_{1}, s, s_{1}^{\prime}\right) Z_{L^{\prime} S^{\prime}}^{J M \epsilon}\left(\Omega_{1}^{\prime}, \Omega_{23}^{\prime}\right),
$$

where a sum is assumed for all repeated indices. The function $Z_{L S}^{J M \epsilon}$ is defined in Eq. 3.

\footnotetext{
${ }^{2}$ At small transferred momentum, the Pomeron trajectory is close to the intercept, which is 1 . The energy behavior $\sim s_{\text {tot }}^{1}$ is then like the one for a vector exchange particle. This explains a dominance of $M=0, M=1$ waves in the COMPASS data.
} 
Due to the orthogonality of the partial-wave basis functions, one obtains the unitarity relation for the partial-wave projected amplitude:

$$
F_{L S}^{J}\left(s, s_{1}\right)-F_{L S}^{* J}\left(s, s_{1}\right)=i \int \mathrm{d} \Phi_{3} T_{L S L^{\prime} S^{\prime}}^{* J}\left(s_{1}, s, s_{1}^{\prime}\right) F_{L^{\prime} S^{\prime}}^{J}\left(s, s_{1}^{\prime}\right) .
$$

The quantities with hat denote amplitudes where the isobar shape is factorized out, $T_{L S L^{\prime} S^{\prime}}^{J}\left(s_{1}^{\prime}, s, s_{1}\right)=$ $f_{S^{\prime}}^{\star}\left(s_{1}^{\prime}\right) \hat{T}_{L S L^{\prime} S^{\prime}}^{J}\left(s_{1}^{\prime}, s, s_{1}\right) f_{S}\left(s_{1}\right)$. To write the unitarity equation for the isobar-reduced amplitude, we decompose the phase space first:

$$
\int \mathrm{d} \Phi_{3} Z_{L^{\prime} S^{\prime}}^{J^{\prime} M^{\prime} \epsilon^{\prime}} f_{S^{\prime}}^{\star}\left(s_{1}\right) Z_{L S}^{J M \epsilon} f_{S}\left(s_{1}\right)=\delta_{J J^{\prime}} \delta_{M M^{\prime}} \delta_{\epsilon \epsilon^{\prime}} \delta_{L L^{\prime}} \delta_{S S^{\prime}} \int \frac{\mathrm{d} s_{1}}{2 \pi} U\left(s_{1}\right) 2 \rho\left(s, s_{1}\right),
$$

where $U\left(s_{1}\right)$ is the isobar-shape function, $U\left(s_{1}\right)=\left|f_{S}\left(s_{1}\right)\right|^{2} \lambda^{1 / 2}\left(s_{1}, m_{\pi}^{2}, m_{\pi}^{2}\right) /(8 \pi s)$. The factor $\rho(s)$ is one half of the two-body phase space. To choose the normalization of $U$, we are guided by the fact that in the Breit-Wigner parametrization the narrow isobar width limit of $f_{S}$ is $(2 \pi) \delta\left(s_{1}-m_{R}^{2}\right)$. Thus we require $\int U\left(s_{1}\right) \mathrm{d} s_{1}=2 \pi$.

The imaginary part of $F_{L S}^{J M \epsilon}$ comes from the presence of singularities. Unitarity cuts are present in both the $s$ and $s_{1}$ complex planes. The discontinuity over the $s_{1}$-unitarity cut gets canceled by the disconnected part of $T$ as shown in Ref. [10]. The discontinuity over the unitarity cut in the $s$ complex plane remains. Denoting $\Delta_{s} Q(s)=Q(s+i \epsilon)-Q(s-i \epsilon)$, we get

$$
\Delta_{S} \hat{F}_{L S}^{J M \epsilon}\left(s, s_{1}\right)=2 i \int \frac{\mathrm{d} s_{1}^{\prime}}{2 \pi} U\left(s_{1}\right) \rho\left(s, s_{1}^{\prime}\right) \hat{T}_{L S L^{\prime} S^{\prime}}^{* J}\left(s_{1}, s, s_{1}^{\prime}\right) \hat{F}_{L^{\prime} S^{\prime}}^{J^{\prime} \epsilon}\left(s, s_{1}^{\prime}\right) .
$$

Simplification 3: the entire $s_{1}$ dependence of $F_{L S}^{J M \epsilon}$ and $T_{L S L^{\prime} S^{\prime}}^{J}$ comes from the isobar parameterization, thus $\hat{F}_{L S}^{J M \epsilon}$ and $\hat{T}_{L S L^{\prime} S^{\prime}}^{J}$ do not depend on $s_{1}$. The proper treatment of three-body unitarity $[10,11]$ includes contributions from cross-channel exchanges in the intermediate state and from real-pion exchange (RPE). We neglect these effects assuming the main contribution to the final state interaction to be from three-body resonances.

\subsection{Parameterization of the scattering amplitude}

The scattering amplitude $T$ is constrained by the equation $\left\langle I^{\prime} \pi\left|S \mathbb{S}^{\dagger}\right| I \pi\right\rangle=(2 \pi)^{4} \delta\left(P-P^{\prime}\right)$. The same steps we have done before for the full-process amplitude are performed now for the scattering amplitude. We arrive at the following equation:

$$
\Delta_{S} \hat{T}_{L S L^{\prime} S^{\prime}}^{J}(s)=2 i \rho_{S^{\prime \prime}}(s) \hat{T}_{L S L^{\prime \prime} S^{\prime \prime}}^{* J}(s) \hat{T}_{L^{\prime \prime} S^{\prime \prime} L^{\prime} S^{\prime}}^{J}(s), \quad \rho_{S}(s)=\int_{4 m_{\pi}^{2}}^{\left(\sqrt{s}-m_{\pi}\right)^{2}} \frac{\mathrm{d} s_{1}}{2 \pi} U\left(s_{1}\right) \rho\left(s, s_{1}\right) .
$$

The expected threshold behavior of the amplitude $F_{L S}^{J M \epsilon}$ is determined by the partial-wave index $L$. The range of interaction is limited by left-hand singularities.For the left singularities, we employ Blatt-Weisskopf [12] type of parameterization.

$$
\hat{T}_{L S L^{\prime} S^{\prime}}^{J}=h_{L} \bar{T}_{L S L^{\prime} S^{\prime}}^{J} h_{L^{\prime}}, \quad h_{L}^{2}=\frac{R^{2} q^{2}}{1+R^{2} q^{2}}, \quad q=4 \pi \rho_{S} \sqrt{s} .
$$

We obtain the final equation for the discontinuity of the fully reduced scattering amplitude across the unitarity cut:

$$
\Delta_{S} \bar{T}_{L S L^{\prime} S^{\prime}}^{J}(s)=2 i \rho_{L^{\prime \prime} S^{\prime \prime}}(s) \bar{T}_{L S L^{\prime \prime} S^{\prime \prime}}^{* J}(s) \bar{T}_{L^{\prime \prime} S^{\prime \prime} L^{\prime} S^{\prime}}^{J}(s), \quad \rho_{L S}=\rho_{S} h_{L}^{2}
$$


Eq. 20 can be satisfied for $T_{L S L^{\prime} S^{\prime}}^{J}$ by construction; this approach is known as the $K$-matrix approach. The latter reads $\bar{T}=K\left[1-i \tilde{\rho}_{L S} K\right]^{-1}$ in a matrix sense with generalized index for $L S$-pair. $\tilde{\rho}_{L S}$ is a dispersive integral, cf. Eq. 21. $K_{L S L^{\prime} S^{\prime}}$ is an arbitrary smooth function which we parametrize by the sum of pole terms.

$$
\tilde{\rho}_{L S}=\frac{1}{\pi i} \int_{s_{\mathrm{th}}}^{\infty} \frac{\rho_{L S}\left(s^{\prime}\right)}{s^{\prime}-s} \mathrm{~d} s^{\prime}, \quad K_{L S L^{\prime} S^{\prime}}=\sum_{R} \frac{g_{L S}^{R} g_{L^{\prime} S^{\prime}}^{R}}{s_{R}-s} .
$$

\subsection{Parameterization of the production amplitude}

The reaction $\pi \mathbb{P} \rightarrow I \pi$ can be considered from the $s$ - and $t$-channel duality point of view. The transferred momentum for the reaction is $t_{1}=\left(p_{A}-p_{2}+p_{3}\right)^{2}$. The range of interaction is determined by $t$-channel exchanges. It is proportional to the mass of the exchange particle. The production of $s$-channel resonances is a short-range process because it can be represented as an infinite sum of $t$-channel exchange diagrams with heavy particles.

The long-range production process is determined by the exchange of the lightest particle. Since pion exchange is allowed in the reaction, the long-range production process becomes very important due to the closeness of the pion pole to the physical region. Essentially, the pion-exchange singularity is the closest left-hand singularity to the physical region, that is why we take it into account explicitly.

Let $\hat{b}_{L S}^{J M \epsilon}$ be the isobar-reduced projection of the pion-exchange diagram to the partial-wave basis. The pion-exchange process in the diffractive production of the $3 \pi$ system is known as the Deckprocess. We use expression (2.12) from Ref. [13] for $b_{L S}^{J M \epsilon}$. A construction which satisfies Eq. 17 and has same left singularities as $b_{L S}^{J M \epsilon}$ has been fond by Omnès [14] and applied to the pion-exchange process by Basdevant and Berger (see Ref. [15]):

$$
\hat{F}_{L S}^{J M \epsilon}=\hat{b}_{L S}^{J M \epsilon}+h_{L} \bar{T}_{L S L^{\prime} S^{\prime}}^{J}\left(c_{L^{\prime} S^{\prime}}+\frac{1}{\pi} \int_{s_{\mathrm{th}}}^{\infty} \frac{\hat{b}_{L^{\prime} S^{\prime}}^{J M \epsilon}\left(s^{\prime}\right) \rho_{S^{\prime}}\left(s^{\prime}\right) h_{L^{\prime}}\left(s^{\prime}\right)}{s^{\prime}-s} \mathrm{~d} s^{\prime}\right),
$$

where the sum over primed indices is assumed. The short-range interaction is parameterized by the $c_{L^{\prime} S^{\prime}}^{J}$, which are smooth functions of $s$, e.g. polynomials in $s$.

The model in Eq. 22 has three components. The first term is the projection of the pion-exchange diagram, the third term is the unitarization of the pion exchange, i.e. when $I \pi$ are produced they keep interacting in a way determined by the scattering matrix. The second term is the short-range resonance production, referred to as a "direct resonance production". Eq. 22 is essentially the model parameterization which has to be fixed by the data.

\section{Pole search}

The amplitude $\bar{T}^{J}$ is an analytic function, defined in the complex $s$-plane. Resonances in the $I \pi$ system can be found by identifying poles in the unphysical Riemann sheets. Every open channel corresponds to a cut starting from the threshold for the corresponding channel. The closest Riemann sheet to the physical region at point $s$ is the one which is introduced by the highest threshold above the point. For the quasi-two-body amplitude of $3 \pi$ scattering, all channels start at $3 \pi$ threshold. Thus, only one unphysical sheet is relevant to the physics along the real axis, the second Riemann sheet.

A complication arises because of the quasi-stable nature of particle $I$ (one can see it as a particle with complex mass). A corresponding unitarity cut appears in the complex plane. One finds the branch point of $\rho_{S}(s)$ due to the singularity in $U\left(s_{1}\right)$ in Eq. 21 . When the $\pi \pi$-amplitude $f_{S}$ is resonant, 
there is a pole of $U\left(s_{1}\right)$ in the complex $s_{1}$-plane, say at $s_{1, R}$. A singularity occurs in the $s$-plane when the upper integration range $\left(\sqrt{s}-m_{\pi}\right)^{2}$ in Eq. 21 arrives at the pole of $U\left(s_{1}\right)$, i.e. $\left(\sqrt{s}-m_{\pi}\right)^{2}=s_{1, R}$ or $s=\left(\sqrt{s_{1, R}}+m_{\pi}\right)^{2}$. The singularity is of the square-root type, as shown in Ref. [16].

The amplitude at the second Riemann sheet can be calculated as the sum of the value of the amplitude at the first Riemann sheet and the discontinuity. The latter has to be found in analytic form. In a single-channel case, it is easier to analytically continue the inverse amplitude because the discontinuity is just given by the phase space. Unfortunately, the amplitude in the coupled-channel case is not necessarily invertible. ${ }^{3} \operatorname{det}[K T]^{-1}(s)$ can be considered as an analytic function to be continued.

$$
\left[K T_{I I}\right]^{-1}=\left[K T_{I}\right]^{-1}-\Delta_{S}[K T]^{-1}, \quad \Delta_{S}[K T]^{-1}=-2 i \rho_{L S} K_{L S L^{\prime} S^{\prime}},
$$

where indices $I, I I$ denote the Riemann sheet with respect to the $3 \pi$ unitarity cut.

Zeros of $\left[K T_{I I}\right]^{-1}$ correspond to poles of $T_{I I}$. The pole positions $\left(m_{R}-i \Gamma_{R} / 2\right)^{2}$ determine the resonance parameters. The pole search procedure is the final step of the analysis.

\section{Application to $J^{P C}=2^{-+}$sector}

The light-quarks meson sector with $J^{P C}=2^{-+}$is an appropriate place to apply the model. The ground state $\pi_{2}(1670)$ decays to $3 \pi$ with $95 \%$ branching ratio, which justifies our elasticity approximation.

The COMPASS PWA model consists of 81 waves with positive reflectivity. 17 waves have $2^{-+}$ quantum numbers. 8 waves contribute to the $J^{P C} M^{\epsilon}=2^{-+} 0^{+}$sector, they are $f_{2} \pi$ S-, D-, H-waves, $\rho \pi \mathrm{P}$-, F-waves, $(\pi \pi)_{S} \pi \mathrm{D}$-wave, $f_{0} \pi \mathrm{D}$-wave, $\rho_{3} \pi \mathrm{P}$-wave.

The partial-wave analysis has been performed independently for several $t^{\prime}$ bins, in the interval from 0.1 to $1 \mathrm{GeV}^{2}$. For different momentum transfer the production parameters might change, e.g. the ratio between short- and long-range production processes. The scattering matrix, in contrast, should not depend on the production mechanism. Thus all data have to be fitted simultaneously using a set of production parameters for every $t^{\prime}$-bin and only one production-independent set of parameters for the scattering matrix.

We show an example application where the five waves of the $2^{-+} 0^{+}$sector with the highest contributions are fitted with the model. The $K$-matrix is parameterized by the sum of 4 poles in the form of Eq. 21. The long-range production has one complex strength parameter for every $t^{\prime}$-bin. The shortrange parameters are five complex numbers for every $t^{\prime}$-bin. The total amount of parameters is 145 . Minimization is done multiple times by sampling different starting points. The Minuit2 package [17] is employed.

\subsection{Fit results}

The COMPASS spin-density matrix $\rho_{L S L S}^{J M \epsilon}=\rho_{L S L S}^{20+}$ for $0.22<t^{\prime}<0.26 \mathrm{GeV}^{2}$ is shown in Fig. 1 . The wave names are indicated in the column and row titles. The argument function is plotted for nondiagonal elements. The fit result of our model is shown by the solid and dashed red lines in the range of the fit and extrapolated outside, respectively. Most of the intensities of the waves are described rather well in the interval of the fit except the $\rho \pi \mathrm{P}$-wave, where the low-mass shoulder is not reproduced by the fit, and the high-mass region in the $(\pi \pi)_{S} \pi \mathrm{D}$-wave. The phases of the interference terms are shown in Fig. 1 as off-diagonal elements.

While the fit generally looks promising, there several issues to be noted.

\footnotetext{
${ }^{3}$ If the $K$-matrix is parameterized by Eq. 21 , the rank of the $T$-matrix is 1 .
} 
- Only two of four poles in the $K$-matrix have converged to values close to the range of the fit. One K-matrix pole runs to negative values of $s$ (see yellow dashed line at $\rho \pi$ F-wave subplot of Fig. 1), the other pole goes to a big positive value giving a smooth rising contribution to the $K$-matrix. To compensate the high values of $s_{R}$ in Eq. 21 , the couplings $g^{R}$ grow. A fit minimum can be found, but it is not well pronounced; there are many local minima.

- The amplitude of the long-range production process drawn by light-green line in Fig. 1 is unexpectedly small. The fit prefers to assign the whole amplitude to the short-range process, likely because this part has more free parameters, i.e. it is more flexible. Another reason might be that the long-range model used is too simplistic and does not reflect the physics right.

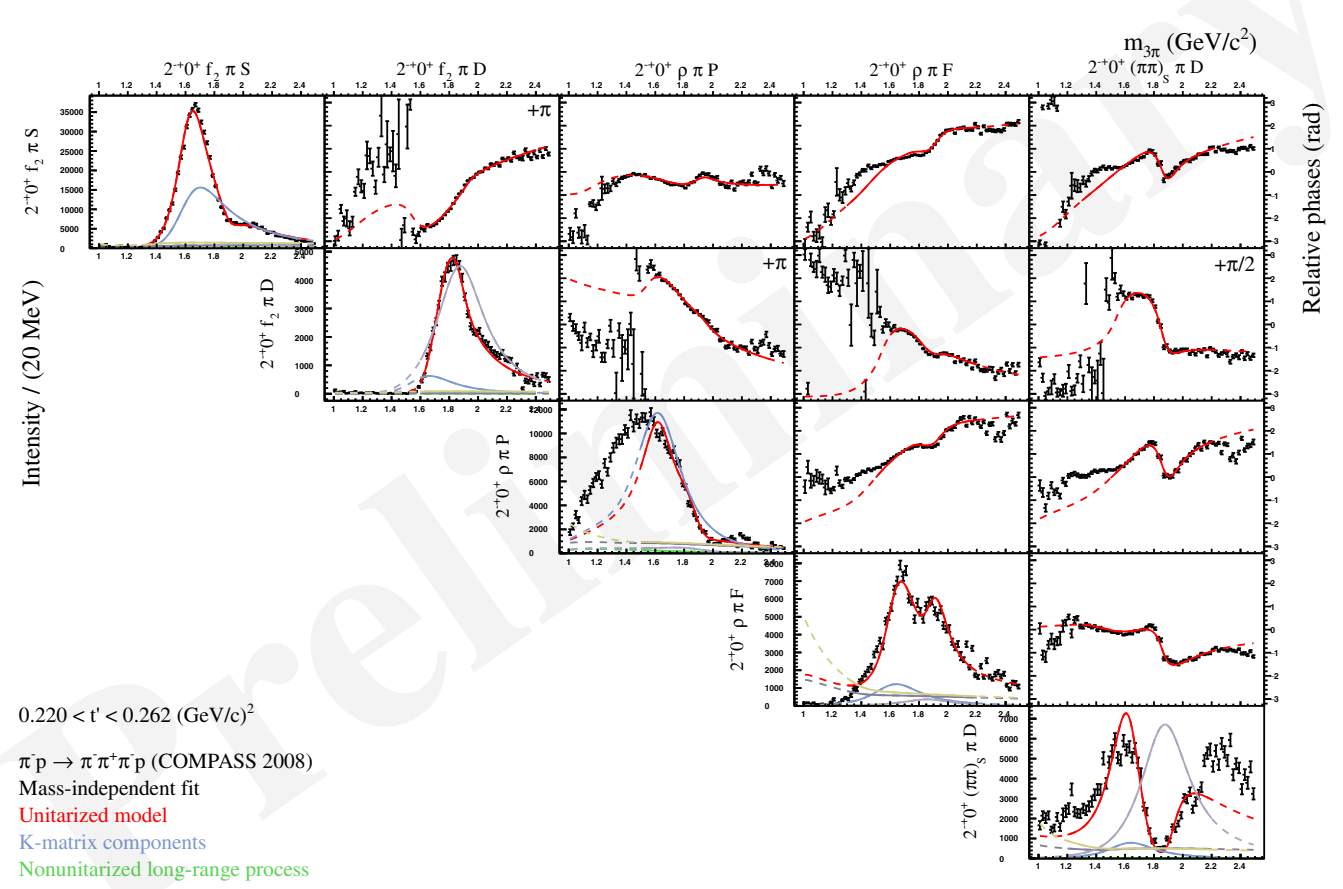

Figure 1. Results of the fit of the COMPASS spin density matrix $\rho_{L S L^{\prime} S^{\prime}}^{20+}$ (see Eq. 8) by the model introduced in the text. The matrix indices run over the following waves: $f_{2} \pi \mathrm{S}$-wave, $f_{2} \pi \mathrm{D}$-wave, $\rho \pi \mathrm{P}$-wave, $\rho \pi \mathrm{F}$-wave, $(\pi \pi)_{S} \pi \mathrm{D}$-wave. The intensities are functions of the invariant mass of $3 \pi$ system. They are plotted along the diagonal. The off-diagonal elements are the phases of the interference terms between different waves. Some of the fitted phases are shifted by a number indicated at the right top side of the subplot for plotting reasons. For the comparison to the data, the developed model is plotted by the red line. The other colored lines indicate the components of the $K$-matrix, i.e. how the amplitude would look like if only one $K$-matrix pole contributed. The range where the model has been fitted is indicated by solid lines, the dashed parts show the extrapolation of the model.

\section{Conclusions}

We have developed a model for the peripheral production of a system of three pseudoscalar mesons. The model is guided by the condition of unitarity. The quasi-two-body unitarity requirements are sat- 
isfied by construction. The interaction of three pions is reduced to an isobar-pion interaction assuming that the main dynamics comes from three-body resonances.

The amplitude for the reaction (1) is expressed through the scattering amplitude $3 \pi \rightarrow 3 \pi$. The production process is considered from a duality point of view. The range of the $\pi \mathbb{P}$ interaction is determined by the mass of the exchange particle. The long-range part is expected to be noticeable due to the pion exchange diagram. The short-range interaction is included to account for all $t$-channel high-mass exchanges.

The scattering amplitude is a unique object which does not depend on the reaction in which the $3 \pi$ final state is produced. A fit of the model to experimental data allows us to fix the parameters of the scattering matrix. Being analytic the amplitude allows to explore the unphysical region attached to the real axis. Poles which cause the resonance behavior of the amplitude can be found. The pole positions and residuals determine the masses, the widths of the resonances and the couplings in an unambiguous way.

COMPASS spin-density matrices from a mass-independent partial-wave analysis are available to apply the model. The power of the data set is the binning in transferred momentum space. The fit is technically challenging. The model used for the $J^{P C} M^{\epsilon}=2^{-+} 0^{+}$discussed in this paper includes 5 coupled channels. It includes over one hundred parameters and is applied to more than one thousand data points. Preliminary results look promising. Systematic studies of the model are ongoing.

\section{References}

[1] J.J. Dudek, R.G. Edwards, C.E. Thomas, D.J. Wilson (Hadron Spectrum), Phys. Rev. Lett. 113, 182001 (2014), 1406.4158

[2] D.J. Wilson, R.A. Briceno, J.J. Dudek, R.G. Edwards, C.E. Thomas, Phys. Rev. D92, 094502 (2015), 1507.02599

[3] A. Jackura, M. Mikhasenko, A. Szczepaniak, EPJ Web Conf. 130, 05008 (2016), 1610.04567

[4] P. Abbon et al. (COMPASS Collaboration), Nucl. Instr. Meth. A 779, 69 (2015), 1410. 1797

[5] C. Adolph et al. (COMPASS Collaboration) (2015), 1509.00992

[6] K. Gottfried, J.D. Jackson, Nuovo Cim. 33, 309 (1964)

[7] M. Jacob, G.C. Wick, Annals Phys. 7, 404 (1959), [Annals Phys.281,774(2000)]

[8] S.U. Chung (1971), CERN-71-08, Lectures given in Academic Training Program of CERN, 1969-1970

[9] J.D. Hansen, G.T. Jones, G. Otter, G. Rudolph, Nucl. Phys. B81, 403 (1974)

[10] G.N. Fleming, Phys. Rev. 135, B551 (1964)

[11] W.J. Holman, Phys. Rev. 138, B1286 (1965)

[12] J.M. Blatt, V.F. Weisskopf, Theoretical Nuclear Physics (Wiley, New York, 1952)

[13] G. Ascoli, L. Jones, B. Weinstein, H. Wyld, Phys. Rev. D 8, 3894 (1973)

[14] R. Omnes, Nuovo Cim. 8, 316 (1958)

[15] J.L. Basdevant, E.L. Berger, Phys. Rev. D16, 657 (1977)

[16] S. Ceci, M. Doring, C. Hanhart, S. Krewald, U.G. Meissner, A. Svarc, Phys. Rev. C84, 015205 (2011), 1104.3490

[17] F. James, M. Roos, Comput. Phys. Commun. 10, 343 (1975) 